\title{
Clinical utility gene card for: Loeys-Dietz syndrome (TGFBR 1/2) and related phenotypes
}

\author{
Mine Arslan-Kirchner ${ }^{\star, 1}$, Jörg T Epplen ${ }^{2}$, Laurence Faivre ${ }^{3}$, Guillaume Jondeau ${ }^{4}$, Jörg Schmidtke ${ }^{1}$, \\ Anne De Paepe ${ }^{5}$ and Bart Loeys ${ }^{5}$
}

European Journal of Human Genetics (2011) 19, doi:10.1038/ejhg.2011.68; published online 27 April 2011

\section{DISEASE CHARACTERISTICS}

1.1 Name of the disease (synonyms)

Loeys-Dietz syndrome (LDS), Marfan syndrome type 2, Marfanrelated phenotype.

\subsection{OMIM\# of the disease}

609192 .

\subsection{Name of the analyzed genes or DNA/chromosome segments TGFBR1/TGFBR2.}

\subsection{OMIM\# of the gene(s)}

190181 and 190182.

\subsection{Mutational spectrum}

As the initial description of the disease, several hundreds of mutations have been found, of which circa $75 \%$ are located in TGFBR2 and circa $25 \%$ in TGFBR1. Both missense, splice site and nonsense mutations have been reported. Most missense mutations occur in the intracellular serine threonine kinase domain of both TGF beta receptors.

\subsection{Analytical methods}

Direct sequencing of all exons with intron/exon boundaries of both genes.

So far no deletions/duplications have been reported after screening by MLPA, so the usefulness of MLPA for testing of the TGFBR1/2 need to be determined (personal communication).

\subsection{Analytical validation}

Sequencing of both strands. When a mutation is identified, confirmation in a second independent DNA sample is recommended.

1.8 Estimated frequency of the disease (incidence at birth ('birth prevalence') or population prevalence)

No data about prevalence have been reported. However, the frequency of mutation in the TGFBR2 gene seems to be more frequent than mutation in the TGFBR1 gene, and both seem to be less frequent than FBN1 mutation.

1.9 If applicable, prevalence in the ethnic group of investigated person

Not applicable.
1.10 Diagnostic setting

\begin{tabular}{lll}
\hline & Yes & No \\
A. (Differential) diagnostics & $\Downarrow$ & $\square$ \\
B. Predictive testing & $\Downarrow$ & $\square$ \\
C. Risk assessment in relatives & $\Downarrow$ & $\square$ \\
D. Prenatal & $\Downarrow$ & $\square$ \\
\hline
\end{tabular}

Comment:

TGFBR $1 / 2$ testing is indicated as the first test to perform in the following indications:

(1) Patients with characteristic triad of LDS: hypertelorism, cleft palate/bifid uvula and aortic/arterial aneurysms/tortuosity. ${ }^{1}$

(2) Patients with aortic/arterial aneurysm (specifically early onset of aortic aneurysm and history of early death) with variable combination of other features including arachnodactyly, camptodactyly, club feet, craniosynostosis (all types), mental retardation, blue sclerae, thin skin with atrophic scars, easy bruising, joint hypermobility, bicuspid aortic valve (BAV) and patent ductus arteriosus (PDA), atrial and ventricular septum defects (ASD/VSD). ${ }^{1}$

(3) Patients with a vascular Ehlers-Danlos like phenotype with normal collagen type III biochemistry, but typical skin findings (thin skin, atrophic scars, easy bruising) and joint hypermobility. ${ }^{2}$

(4) Patients with Marfan-like phenotype, especially those without ectopia lentis but with aortic/arterial aneurysms, craniofacial features (malar hypoplasia and retrognathia) and skeletal features not fulfilling Ghent criteria for MFS (arachnodactyly, pectus deformities, scoliosis, joint laxity and dolichostenomelia). ${ }^{2,3}$

(5) Families with autosomal dominant thoracic aortic aneurysms, especially those families with precocious aortic/arterial dissection, aortic disease beyond the aortic root (including cerebral arteries), aortic/arterial tortuosity, association with ASD/VDS/ PDA. Mild marfanoid skeletal features may be present. ${ }^{4,5}$

TGFBR $1 / 2$ testing is also indicated as a secondary test in patients in whom FBN1 testing remains negative despite MFS-like phenotype.

TGFBR1/2 mutations have been described in Shprintzen-Goldberg, but most of these patients have many characteristics in common with

\footnotetext{
${ }^{1}$ Institute of Human Genetics, Hannover Medical School, Hannover, Germany; ${ }^{2}$ Human Genetics Bochum, Ruhr University Bochum, Bochum, Germany; ${ }^{3}$ Centre de Génétique, Centre Hospitalier Universitaire de Dijon, Dijon, France; ${ }^{4}$ Service de Cardiologie, CHU Hôpital Xavier Bichat - Claude Bernard, Paris, France; ${ }^{5}$ Center for Medical Genetics, Ghent University, Ghent, Belgium

*Correspondence: Dr M Arslan-Kirchner, Institute of Human Genetics, Hannover Medical School, Carl-Neuberg-Str. 1, Hannover D-30625, Germany. Tel: +49 511 532 6532;

Fax: +49 511532 8533; E-mail: arslan.mine@mh-hannover.de
} 
LDS. Many patients with true Shprintzen-Goldberg syndrome do not have TGFBR1/2 mutations. ${ }^{1,6}$

Although initially two clinical presentations were distinguished (LDS type I with more typical craniofacial findings and LDS type II with more prominent cutaneous findings), it is now recognized that LDS is a continuum that includes a wide range of clinical presentations. The respective clinical spectrum of TGFBR1 and TGFBR2 mutation is not fully recognized.

\section{TEST CHARACTERISTICS}

\begin{tabular}{|c|c|c|c|c|}
\hline & \multicolumn{2}{|c|}{ Genotype or disease } & \multirow{2}{*}{$\begin{array}{l}\text { A: True positives } \\
\text { B: False positives }\end{array}$} & \multirow{2}{*}{$\begin{array}{l}\text { C: False negative } \\
\text { D: True negative }\end{array}$} \\
\hline & Present & Absent & & \\
\hline \multicolumn{5}{|l|}{ Test } \\
\hline \multirow[t]{2}{*}{ Positive } & A & B & Sensitivity: & $A /(A+C)$ \\
\hline & & & Specificity: & $D /(D+B)$ \\
\hline \multirow[t]{2}{*}{ Negative } & C & D & Positive predictive value: & $A /(A+B)$ \\
\hline & & & Negative predictive value: & $\mathrm{D} /(\mathrm{C}+\mathrm{D})$ \\
\hline
\end{tabular}

\subsection{Analytical sensitivity}

(proportion of positive tests if the genotype is present)

Nearly $100 \%$.

The possibility of preferential amplification of one allele, if primers are localized on a SNP (hampering primer binding) or because of deletion (making amplification impossible) exists, although these events are exceptional.

Classical criteria for determining the pathogenicity of a mutation are the following:

- Nonsense mutation

- Splice site mutation affecting canonical splice sequence or shown to alter splicing on mRNA/cDNA level

- Out of frame and inframe deletion/insertion

- De novo missense mutation (with proven paternity and absence of disease in parents)

- Missense mutation previously been shown to segregate in Loeys-Dietz family

- For other missense mutations, the segregation in family should be checked, if possible, as well as the absence of the variant in 400 ethnically matched control chromosomes.

\subsection{Analytical specificity}

(proportion of negative tests if the genotype is not present)

Nearly $100 \%$.

\subsection{Clinical sensitivity}

(proportion of positive tests if the disease is present)

The clinical sensitivity can be dependent on variable factors such as age or family history. In such cases a general statement should be given, even if a quantification can only be made case by case.

Unknown, dependent on the depth of clinical evaluation and cardiovascular imaging.

\subsection{Clinical specificity}

(proportion of negative tests if the disease is not present)

The clinical specificity can be dependent on variable factors such as age or family history. In such cases a general statement should be given, even if a quantification can only be made case by case.
By definition $100 \%$.

Mutations within the TGFBR1 and TGFBR2 genes have been reported in patients with LDS., ${ }^{1,2}$

TGFBR1/2 mutations have also been found in MFS or suspected MFS. ${ }^{3}$ Sakai et $a l^{7}$ found one patient with a TGFBR1 mutation (2\%) and two TGFBR2 mutations (4\%) out of a series of 49 MFS-like patients; Màtyàs et al ${ }^{8}$ reported 10 TGFBR1 or TGFBR2 mutations in 70 unrelated individuals with MFS-like phenotypes who previously tested negative for mutations in FBN1; Singh et al ${ }^{9}$ found two TGFBR1 and five TGFBR2 mutations in 41 unrelated patients who did or did not fulfill the diagnostic criteria for MFS, in whom mutations in the FBN1-coding region were not identified; Stheneur et $a l^{10}$ found six mutations in the TGFBR2 gene and one in the TGFBR1 gene in 105 MFS patients, and nine mutations in the TGFBR2 gene and two mutations in the TGFBR1 gene in 247 patients with incomplete or probable MFS that were negative for a FBN1 gene mutation. Chung et $a l^{11}$ found six TGFBR2 mutations in 41 MFS-like probands (previously negative for FBN1). None of these six patients with TGFBR2 mutations fulfilled the clinical MFS criteria from the Ghent nosology.

TGFBR1/2 mutations have been described in familial thoracic aortic aneurysm families, ${ }^{4,5}$ but many of these families have not been evaluated in detail for clinical features that are common in LDS.

Finally, as stated above, some mutations have been described in Shprintzen-Goldberg syndrome, but these patients have features that are believed to be more common in LDS. $^{6}$

No TGFBR1/2 mutations have been found in families with presentation of isolated bicuspid aortic valve with or without aortic dilatation. $^{12}$

\subsection{Positive clinical predictive value}

(life-time risk to develop the disease if the test is positive)

Nearly $100 \%$.

Incomplete penetrance, ${ }^{1}$ as well as somatic mosaicism ${ }^{13}$ have been reported.

\subsection{Negative clinical predictive value}

(probability not to develop the disease if the test is negative)

Assume an increased risk based on family history for a non-affected person. Allelic and locus heterogeneity may need to be considered.

Index case in that family had been tested:

Nearly $100 \%$.

Index case in that family had not been tested:

Predictive testing for family members should only be proposed, if a pathogenic mutation has been identified in an index case.

\section{CLINICAL UTILITY}

3.1 (Differential) diagnosis: The tested person is clinically affected (To be answered if in 1.10 'A' was marked)

\subsubsection{Can a diagnosis be made other than through a genetic test?}

\begin{tabular}{lll}
\hline No & $\square$ (continue with 3.1.4) & \\
Yes & $\bigotimes$ & $\square$ \\
& Clinically & $\square$ \\
Imaging & $\square$ \\
Endoscopy & $\square$ \\
Biochemistry & $\square$ \\
Electrophysiology & Autopsy and histological verification of \\
Other (please describe) & the typical findings are reasonable. \\
&
\end{tabular}


3.1.2 Describe the burden of alternative diagnostic methods to the patient

Physical exam, cardiological (including echocardiography) and vascular investigations (3D-CTscan or MR angiography) can altogether establish a diagnosis (but not always). These investigations are always necessary to evaluate the phenotype of the patient, which indicates the severity of the prognosis and guide therapy.

3.1.3 How is the cost effectiveness of alternative diagnostic methods to be judged?

Unknown.

3.1.4 Will disease management be influenced by the result of a genetic test?

No $\square$

Yes $\otimes$

Therapy Regular and frequent cardiovascular follow-up and early surgery (please with aortic root replacement are mandatory. For young children

describe) with severe systemic findings of LDS, surgical repair of the ascending aorta should be considered early once the maximal dimension allows the placement of a graft of sufficient size to accommodate growth. Timing for surgery will always depend not only on the presence of TGFBR mutation, but also on the severity of the phenotype and family history. For adolescents and adults severely affected, some authors recommend surgical repair of the ascending aorta to be considered earlier than in classical MFS, such as an aortic diameter of $40 \mathrm{~mm}^{2}$. This recommendation is based on examples of documented aortic dissection in adults with aortic root dimensions at or below $4.0 \mathrm{~cm}$ and the excellent response to prophylactic surgery. However, series report excellent prognosis when aortic root surgery is carried out above $50 \mathrm{~mm}$, in adult patients presenting less severe phenotype. ${ }^{14}$ During pregnancy there is a risk for visceral rupture, ante- or post-partum hemorrhage and poor wound healing. Frequent monitoring of the aorta is essential for pregnant woman, and cervical instability should be excluded in the event that a general anaesthetic and caesarean section is required. Referral to a high-risk obstetric management clinic and delivery in a large tertiary referral centre is recommended. Prenatal diagnosis or, in some countries, preimplantation genetic diagnosis is possible, if the disease causing mutation is known.

Prognosis There is evidence that patients with TGFBR1/2 mutation need (please more extensive imaging of the aorta, and in some series, have describe) increased risk for dissection at smaller aortic diameters than patients with classic MFS. ${ }^{2}$ Therefore, identification of TGFBR1/2 mutation could influence prognosis.

Management Management should be coordinated in multidisciplinary clinic, (please experienced in the management of aortic aneurysm syndromes.

describe) Important considerations when managing cardiovascular features of Loeys-Dietz syndrome are:

- Aortic dissection may occur at smaller aortic diameters than observed in Marfan syndrome in severe phenotype

Vascular disease may not be limited to the aortic root

- Beta-adrenergic blockers or other medications are used to reduce hemodynamic stress

- Aneurysms are amenable to surgical intervention.

Surgical fixation of cervical spine instability may be necessary to prevent spinal cord damage. Clubfeet and severe pes planus are treated in by standard protocols. Treatment of cleft palate and craniosynostosis is by standard protocols; management by a craniofacial team is preferred.
3.2 Predictive setting: The tested person is clinically unaffected but carries an increased risk based on family history

(To be answered if in 1.10 ' $\mathrm{B}$ ' was marked)

\subsubsection{Will the result of a genetic test influence lifestyle and prevention?}

If the test result is positive (please describe):

Yes.

All individuals with LDS require echocardiography at frequent intervals to monitor the status of the ascending aorta (usually yearly, but depending on the severity of the phenotype); the frequency of magnetic resonance angiography (MRA) or computerized tomography angiography (CTA) evaluations depend on clinical findings. Individuals with cervical spine instability and severe or progressive scoliosis should be followed by an orthopedic surgeon.

Contact sports, competitive sports, isometric exercise, agents that stimulate the cardiovascular system including routine use of decongestants should be avoided, as well as activities that cause joint injury or pain

If the test result is negative (please describe):

Follow-up is dispensable and restriction of sports is unnecessary, if a familial mutation can be excluded. A negative result should be interpreted with care, if the mutation is unknown in the index patient. Testing for mutation in other genes should be considered, depending on the phenotype (FBN1, ACTA2 and MYH11).

3.2.2 Which options in view of lifestyle and prevention does a person at-risk have if no genetic test has been done (please describe)?

Regular clinical follow-up similar to those with a mutation identified. Limitation of sports, beta-blockade are indicated depending on the confidence of the clinical diagnosis or evaluated aortic risk.

3.3 Genetic risk assessment in family members of a diseased person (To be answered if in 1.10 ' $\mathrm{C}$ ' was marked)

3.3.1 Does the result of a genetic test resolve the genetic situation in that family?

Yes.

3.3.2 Can a genetic test in the index patient save genetic or other tests in family members?

Yes.

3.3.3 Does a positive genetic test result in the index patient enable a predictive test in a family member?

Yes.

\subsection{Prenatal diagnosis}

(To be answered if in 1.10 ' $\mathrm{D}$ ' was marked)

3.4.1 Does a positive genetic test result in the index patient enable a prenatal diagnostic?

Yes.

\section{IF APPLICABLE, FURTHER CONSEQUENCES OF TESTING}

Please assume that the result of a genetic test has no immediate medical consequences. Is there any evidence that a genetic test is nevertheless useful for the patient or his/her relatives? (Please describe)

Yes, other diagnostic testing becomes unnecessary. Patients and parents of affected children are usually relieved that the disease has been identified ('received a name'). They can seek contact with other persons affected by this disease through patient organization, which is usually seen as an enormous help in coping with the condition. 
Genetic testing also allows early recognition of affected members who may benefit from preventive medical/surgical care due to wide intrafamilial variability.

Lastly, a negative test is very useful for the patient and their children as it removes the aortic threat, and abolishes needs for medical care and follow-up.

\section{CONFLICT OF INTEREST}

The authors declare no conflict of interest.

\section{ACKNOWLEDGEMENTS}

This work was supported by EuroGentest, an EU-FP6 supported NoE, contract number 512148 (EuroGentest Unit 3: 'Clinical genetics, community genetics and public health', Workpackage 3.2).

1 Loeys BL, Chen J, Neptune ER et al: A syndrome of altered cardiovascular, craniofacial, neurocognitive and skeletal development caused by mutations in TGFBR1 or TGFBR2. Nat Genet 2005; 37: 275-281.

2 Loeys BL, Schwarze U, Holm T et al: Aneurysm syndromes caused by mutations in the TGF-beta receptor. N Engl J Med 2006; 355: 788-798.

3 Mizuguchi T, Collod-Beroud G, Akiyama T et al: Heterozygous TGFBR2 mutations in Marfan syndrome. Nat Genet 2004; 36: 855-860.

4 Pannu H, Fadulu VT, Chang J et al: Mutations in transforming growth factor-beta receptor type II cause familial thoracic aortic aneurysms and dissections. Circulation 2005; 112: 513-520.
5 Tran-Fadulu VT, Pannu H, Kim DH et al: Analysis of multigenerational families with thoracic aortic aneurysms and dissections due to TGFBR1 or TGFBR2 mutations. J Med Genet 2009.

6 Robinson PN, Neumann LM, Tinschert S: Response to Kosaki et al 'Molecular pathology of Shprintzen-Goldberg syndrome'. Am J Med Genet 2006; 140A: 109-110.

7 Sakai $\mathrm{H}$, Visser R, Ikegawa $\mathrm{S}$ et al: Comprehensive genetic analysis of relevant four genes in 49 patients with Marfan syndrome or Marfan-related phenotypes. Am J Med Genet A 2006; 140: 1719-1725.

8 Matyas G, Arnold E, Carrel T et al: Identification and in silico analyses of novel TGFBR1 and TGFBR2 mutations in Marfan syndrome-related disorders. Hum Mutat 2006; 27: 760-769.

9 Singh KK, Rommel K, Mishra A et al: TGFBR1 and TGFBR2 mutations in patients with features of Marfan syndrome and Loeys-Dietz syndrome. Hum Mutat 2006; 27: 770-777.

10 Stheneur C, Collod-Beroud G, Faivre L et al: Identification of 23 TGFBR2 and 6 TGFBR1 gene mutations and genotype-phenotype investigations in 457 patients with Marfan syndrome type I and II, Loeys-Dietz syndrome and related disorders. Hum Mutat 2008; 29: E284-E295.

11 Chung BH, Lam ST, Tong TM et al: Identification of novel FBN1 and TGFBR2 mutations in 65 probands with Marfan syndrome or Marfan-like phenotypes. Am J Med Genet A 2009; 149A: 1452-1459.

12 Arrington $\mathrm{CB}$, Sower $\mathrm{CT}$, Chuckwuk $\mathrm{N}$ et al: Absence of TGFBR1 and TGFBR2 mutations in patients with bicuspid aortic valve and aortic dilation. Am J Cardiol 2008; 102: 629-631.

13 Watanabe Y, Sakai H, Nishimura A et al: Paternal somatic mosaicism of a TGFBR2 mutation transmitting to an affected son with Loeys-Dietz syndrome. Am J Med Genet $A$ 2008; 146A: 3070-3074.

14 Attias D, Stheneur C, Roy C et al: Comparison of clinical presentations and outcomes between patients with TGFBR2 and FBN1 mutations in Marfan syndrome and related disorders. Circulation 2009; 120: 2541-2549. 